\title{
Rupture of left ventricle after mitral valve repair: case report and new technique of repair
}

\author{
Mohammad B Izzat, Geoffrey H Smith
}

\begin{abstract}
Type 1 rupture of the left ventricle occurred during mitral valve repair. A repair operation, in which the posterior leaflet of the mitral valve was used as a buttress, was successful.
\end{abstract}

(Br Heart $\mathcal{F} 1993 ; 69: 366-367)$

Rupture of the left ventricle is one of the major life threatening complications of mitral valve surgery, usually after the prosthetic replacement of the mitral valve. We report a case of rupture of the left ventricle occurring after mitral valve repair. This was repaired successfully.

\section{Case report}

A 63 year old woman with longstanding mitral regurgitation and atrial fibrillation was referred for mitral valve surgery. Cardiac catheterisation had shown good left ventricular function, severe mitral regurgitation, and a very large left atrium. The aortic and tricuspid valves and coronary arteries seemed normal.

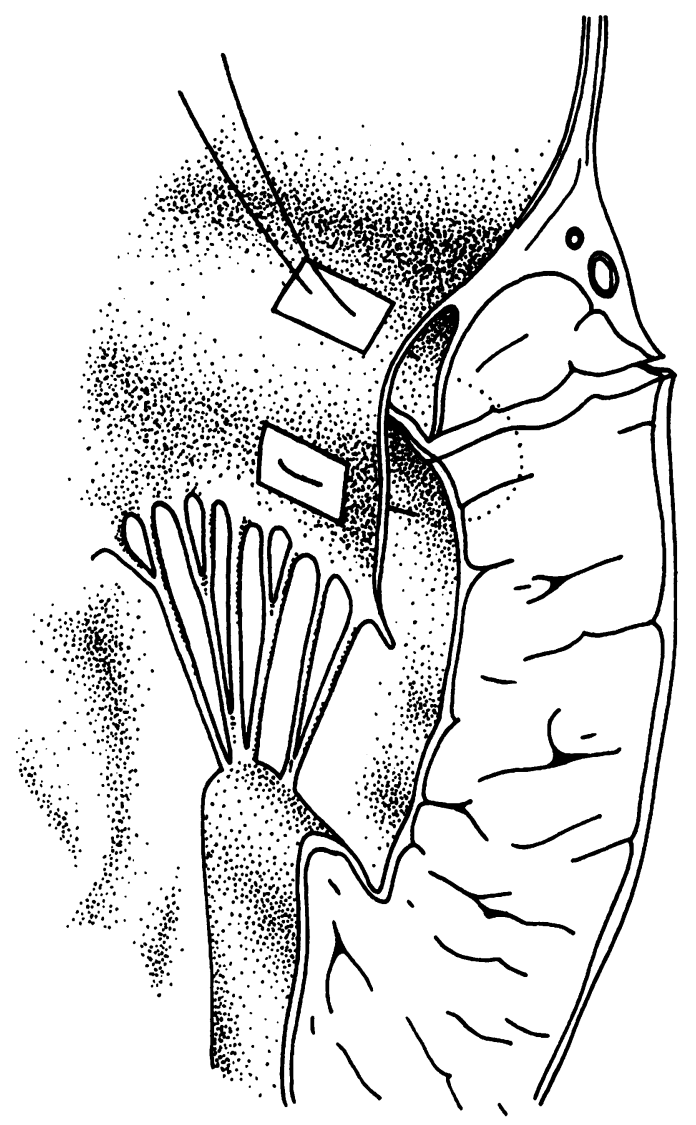

Technique of repair of type 1 rupture of left ventricle with posterior leaflet of the mitral valve.
The operation was performed with car- $\overrightarrow{\vec{D}}$ diopulmonary bypass and cold blood cardio- $\overline{0}$ plegia. No left ventricular apical vent was used. The left atrium was opened to expose $\frac{\bar{m}}{\square}$ the mitral valve, which was found to have $\mathbb{\Phi}$ myxomatous degeneration. There were no major lesions or calcifications in the cusps.

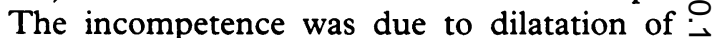
the ring. A Carpentier-Edwards Ring (Model $\vec{\omega}$ 4400 , Baxter) size $30 \mathrm{~mm}$ was inserted with interrupted sutures. This produced a totally competent valve in intraoperative tests by 8 saline injection into the left ventricle. The left atrium was repaired and the heart de-aired.

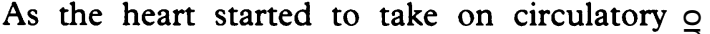
support, severe bleeding occurred from the $\rightarrow$ back of the heart and exploration of the atrio- $D$ ventricular sulcus showed bleeding from the atrioventricular groove close to the left atrial $\vec{\bullet}$ appendage. An attempt was made to repair $\stackrel{\oplus}{\omega}$ this externally but it was ineffective.

Bypass was reinstituted and the heart stopped by further cold blood cardioplegia. The left atrium was reopened to expose the repair, which still seemed to be satisfactory with no evidence of rupture involving the atrium. The ring was removed and the anterior mitral leaflet was excised. A $1 \mathrm{~cm}$ long tear was found $0.5 \mathrm{~cm}$ below and parallel to the atrioventricular ring at about four o'clock. Using the posterior leaflet of the mitral valve we repaired the defect with buttressed sutures (fig). The mitral valve was replaced with interrupted buttressed sutures. A $25 \mathrm{~mm}$ Carbomedics valve was used. The heart came off bypass easily with no bleeding problems.

After operation, the patient required tem- $D$ porary pacing due to bradycardia. An echo- 으․ cardiogram was performed. This showed $\bar{N}$ good function of the prosthetic valve. The patient was discharged home on the 14th postoperative day. She was reviewed as an outpatient eight weeks later and no clinical evidence of valve malfunction was found.

\section{Discussion}

Rupture of the left ventricle is now seen less often than 15 to 20 years ago. None the less, the persisting $75 \%$ mortality makes welcome any new insight into its prevention and management. This entity was first described by Roberts and Morrow in 1967.' Since then about 150 cases have been reported ${ }^{2}$ and $\underset{\rightleftharpoons}{\rightleftharpoons}$ there have been many others unreported. We are not aware of any reported case occurring after mitral valve repair. The surgeon who performed this operation is experienced in mitral valve repair and has not encountered a similar case before. ${ }^{3}$ 
Rupture of the left ventricle is classified into three types based on the location of the epicardial tear. ${ }^{45}$ Our patient had type 1 lesion, which is a defect along the posterior atriventricular sulcus.

Detailed analysis of the available information on our patient showed no specific cause for her ventricular rupture. The analysis of the collected experience suggests that three influences together are necessary to produce the cardiac lesion. These are predisposition by an underlying weakened myocardium, factors that initiate the primary tear, and dynamic forces that contribute to the formation of the primary tear or a covert partial thickness injury into a transmural rupture. ${ }^{6}$

PREDISPOSING FACTORS

Advanced age, ${ }^{5}$ female sex, small body size, and a small sized left ventricle have been mentioned as predisposing factors; all presumably reflect a weakened myocardium and enhanced vulnerability to the injury. ${ }^{46}$ Cardioplegia has also been implicated through increased susceptibility to overdistention of a poorly contracting ventricle from the residual effects of cardioplegia. A few of these factors may apply to our patient but not one of these theories is supported by convincing evidence. ${ }^{6}$

\section{PRODUCTION OF PRIMARY TEAR}

Resection of excessive tissue, deep placement of sutures that fix the valve, insertion of too large a prosthesis, tears at the site of adhesions from a previous operation, and stretch injury due to an untethered left ventricular loop ${ }^{6}$ have all been blamed for left ventricular rupture but do not apply to our case. Forceful traction on the annulus, left ventricular apical venting, and distortion of the left ventricle by lifting, ${ }^{56}$ compressing the ventricle against a rigid prosthesis, and damage due to a metal vent, scissors, and rigid sucker have been proposed as possible causes. ${ }^{6}$ Some might possibly apply to this case.

Beyond the tangible correctable factors, there may still be an occasional patient whose rupture is secondary to problems beyond the surgeon's control. These include rheumatic transmural fibrosis in the area posterolateral to the valve annulus (an area of inherent developmental weakness), and unusual factors such as myocardial abscess. ${ }^{2}$

\section{REPAIR TECHNIQUE}

The basic requirements for successful repair of the tear are closure of the full extent of the tear, placement of sutures into healthy myocardium and not into the injured oedematous tissue in the immediate vicinity of the tear, and (especially in type 1) sparing the left circumflex coronary artery and its major marginal branches.

In type 1 defects, the site of actual perforation can be distant from the site of obvious haemorrhage because the atrioventricular haematoma often disguises the actual defect. In our patient the external epicardial tear was at nine o'clock whereas the internal tear was at four o'clock. Repair should be accomplished with the aid of cardiopulmonary bypass on a decompressed and arrested heart, and it is best performed by an internal approach through left atriotomy. The suture repair may occlude the main circumflex coronary artery. This is more likely with external than with internal correction but it can happen with either technique. The surgeon must be prepared to insert a bypass graft if myocardial ischaemia is suspected in the area supplied by the circumflex artery after completion of the repair. Several successes have been reported with regional revascularisation. ${ }^{6}$

Lennox compressed type 1 tears with buttressed mattress sutures between the left ventricle and the atrium. ${ }^{7}$ In the atrioventricular groove, the sutures were passed either through the sewing ring of the prosthesis or slightly more superficially but under the circumflex artery. Devineni and McKenzie used the left atrial appendage to cover a type 1 rupture, and sutured it to the left ventricular wall below the defect. ${ }^{8}$ The method of Treasure and associates consists of placing mattress sutures from the sewing ring of the prosthesis through the ventricle below the tear. ${ }^{4}$ The sutures are tied on the epicardial surface over Teflon felt patches.

The figure shows our technique, we used the posterior leaflet itself as a buttress. This helped in (a) maintaining complete closure of the defect, $(b)$ avoiding any injury to the circumflex artery, $(c)$ ensuring that the longtudinal loop of the left ventricle is left intact. Lillehei and co-workers reported that ventricular function was improved after operation when the papillary muscles and chordae tendineae were preserved in mitral valve replacement. ${ }^{9}$

The increased tendency to use bi-leaflet prosthetic valves together with preservation of the posterior leaflet in mitral valve replacements make the use of our technique possible and advantageous in repair of left ventricular rupture after mitral valve replacement.

1 Roberts WC, Morrow AG. Cause of early postoperative death following cardiac valve replacement. $\mathcal{f}$ Thorac Cardiovasc Surg 1967;54:422-37.

2 Rainer WG. Rupture of left ventricle following mitral valve replacement. Ann Thorac Surg 1988;46:488.

3 Cooper GJ, Wright EM, Smith GH. Mitral valve repair: a valuable procedure with good long term results even valuable procedure with good long term results even
when performed infrequently. Br Hear $f$ 1991;

4 Treasure Ri, Rainer WG, Streavey TE, Sadler TR. Intraoperative left ventricular rupture associated with mitral valve replacement. Chest 1974;66:511-4.

5 Miller DW Jr, Johnson DD, Ivey TD. Does preservation of the posterior chordae tendineae enhance survival during mitral valve replacement? Ann Thorac Surg 1979; 28:22-7.

6 Karlson KJ, Ashraf MM, Berger RL. Rupture of left ventricle following mitral valve replacement. Ann Thorac Surg 1988;46:590-7.

7 Spencer FC, Galloway AC, Colvin SB. Clinical evaluation of the hypothesis that rupture of the left ventricle following mitral valve replacement can be prevented by lowing mitral valve replacement can be prevented by preservation of the
Surg 1985;6:673-80.

8 Devineni R, McKenzie FN. Type 1 left ventricular rupture after mitral valve replacement. $\mathcal{I}$ Thorac Cardiovasc Surg 1983;86:742-5.

9 Lillehei CW, Kevy MJ, Bonnabeau RC Jr. Mitral valve replacement with preservations of papillary muscles and
chordae tendineae. 7 Thorac Cardiovasc Surg 1964;47: 532-43. 